\title{
Editorial \\ Special issue on control of complex systems
}

Nowadays control systems can find applications in many areas, like aerospace, motion tracking, chemical engineering, physics, biology, economics. To improve control performance is a perpetual goal. With recent progresses of computing technologies, better control performance can be achieved by more judicious control strategies based on more precise and more complicated, such as time-varying, nonlinear, models.

Another trend to improve control performance is built upon the divide-and-conquer philosophy, i.e., a complicated control task is cooperatively accomplished by multiple controllers/agents, instead of a single super-powerful controller. This trend is made possible due to great advances in communication, which enable the information exchange among agents and may unite the less powerful agents. The cost for such control performance improvement is higher spatial complexity of control systems.

Although high model complexity and spatial complexity can be physically handled by current powerful controllers/agents, the lack of efficient analysis and synthesis methods prevents the performance improvement of control systems with high model or/and spatial complexity. As we know, conventional control methods are mainly developed under the assumptions of linear, time-invariant and centralized models. In order to resolve the issues due to the aforementioned two types of complexities, new methods are expected, which is exactly the major aim of the present special issue.

Specifically, the following contributions have been included in this special issue.

1) The contribution by Haghighi and Pang presents an energy-efficient method for distributed region formation flying of nanosatellites. Its concerned framework consists of concurrent estimation and formation sub-schemes. A distributed estimator is developed for the estimation sub-scheme while the formation sub-scheme is handled by an optimal region following formation method based on the receding horizon control (RHC) using the estimated reference orbital elements. Simulations were done to verify the performance improvement of the proposed method.

2) The contribution by Liu and Huang studies the leader-following consensus problem for a class of second-order nonlinear multi-agent systems subject to linearly parameterized uncertainty and disturbance. The problem is solved by integrating the adaptive control technique and the adaptive distributed observer method. The design procedure is further illustrated by an example with a group of Van der Pol oscillators as the followers and a harmonic system as the leader.

3) The contribution by Shi et al. presents a new optimized decentralized controller design method for solving the tracking and disturbance rejection problems for large-scale linear time-invariant systems, using only low-order decentralized controllers. To illustrate the effectiveness of the proposed method, the control of a large flexible space structure is studied and compared with the standard centralized LQR-observer controller. The order of the resultant decentralized controller is much lower than that of the standard centralized LQR-observer controller. The proposed controller also has certain fail-safe properties and, in addition, can be five orders of magnitude more robust than the standard LQR-observer controller based on their real stability radii. The new decentralized controller design method is applied to a large flexible space structure system with 5 inputs and 5 outputs and of order 24.

4) The contribution by Senel and Akar studies the performance of a distributed power adjustment algorithm for shared and split spectrum allocation setups. Its theoretical analysis reveals that the exponential convergence of the power control algorithm can be guaranteed under different spectrum allocation schemes. Its performance analysis

(c) 2016 South China University of Technology, Academy of Mathematics and Systems Science, CAS, and Springer-Verlag Berlin Heidelberg 
is also carried out via simulations which demonstrate the algorithm's fairness under Jain's and Atkinkons's fairness indices.

5) The contribution by George and Subramanian considers the adaptive control of nonlinear systems that are linear in the unknown but time-varying parameters. To achieve satisfactory transient performance, it takes multiple models to respond to the abrupt changes of parameters. More specifically, it considers both the multiple models with switching and tuning methodology as well as multiple models with second level adaptation for this class of systems. The latter approach is demonstrated to be better than the former.

6) The contribution by Zheng et al. considers the consensus problem of a group of homogeneous agents. These agents are governed by a general linear system and can only directly measure the output, instead of the state. It investigates the consensus performance optimization with respect to the common state observation gain and control gain of all agents. In order to resolve the issue of the bilinear structure of the original optimization, it introduces a perturbation method to transform the original bilinear optimization into a series of linear matrix inequality optimizations which can be iteratively solved to greatly improve the consensus performance. Simulations are done to demonstrate the effectiveness of the proposed method.

7) The contribution by Venkatesh proposes some new frequency-domain criteria for the $L_{2}$-stability of both nonlinear single-input-single-output (SISO) and nonlinear multiple-input-multiple-output (MIMO) feedback systems, described by nonlinear integral equations. The stability criteria are derived from some recently introduced algebraic inequalities concerning the scalar and vector nonlinearities, and involve the causal+anticausal O'Shea-Zames-Falb multiplier function (scalar for SISO systems and matrix for MIMO systems). The stability criteria, which are independent of Lyapunov- Krasovskii or Lyapunov-Razumikhin functions and do not seem to be derivable by invoking linear matrix inequalities, seem to be the first of their kind. Two numerical examples for each of SISO and MIMO systems illustrate the criteria.

8) The contribution by Jáuregui et al. presents some simulation and experimental results on the conical tank level control. It studies and compares PI/PID controllers of integer order (IO) as well as of fractional order (FO). The tuning parameters are obtained first by using root locus (RL) and Ziegler and Nichols methods, for comparison purposes. Next, particle swarm optimization (PSO) is employed to determine the optimal controllers' parameters using as fitness function the integral of the absolute value of tracking error (IAE). The experimental results confirm that $\mathrm{PI} / \mathrm{FOPI}$ are the controllers presenting the lowest IAE indexes, whereas PID/FOPID controllers present the lowest energy consumption by the control signal.

Among the abundant research results regarding complex control systems, only a few ones are introduced here. We hope this issue may shed light on developing novel methods to improve the control performance of systems with high model complexity or/and high spatial complexity.

We would like to thank all the people who contributed in some way to this issue. The excellent works by all authors are fundamental for this issue. Without the careful and invaluable revision suggestions, this special issue could not have been accomplished. Special thanks to Prof. Yiguang Hong for deciding to dedicate a special issue to this complex control topic.

\section{Guest editors:}

Hai Lin

Dept. Electrical Engineering, University of Notre Dame

Notre Dame, IN 46556, U.S.A.

E-mail: hlin1@nd.edu

Cheng Xiang

Dept. Electrical and Computer Engineering, National University of Singapore

Singapore 119260

E-mail: elexc@nus.edu.sg

Qiang Ling

Dept. Automation, University of Science and Technology of China

Hefei Anhui 230027, China

E-mail: qling@ustc.edu.cn 